\title{
Stealth Performance Measurement for Radar Targets Based on Railborne Pseudo- Turntable Imaging
}

\author{
Weijie Yang, Xiongjun Fu*, Lin Wang, Ting Li, Meiguo Gao \\ Radar Research Laboratory,Beijing Institute of Technology, Beijing, P.R.China \\ fuxiongjun@bit.edu.cn
}

\begin{abstract}
Stealth performance is an important factor to concern in modern aircrafts design. How to measure the targets' radar stealth performance so as to guide the implementation of RCS reduction measures effectively, has always been a hot issue in the field of electromagnetism. This paper proposes a method of stealth performance measurement for radar targets based on railborne pseudo-turntable imaging, and describes the echo processing algorithm in details. The validation of this method is demonstrated by simulation experiments.
\end{abstract}

Keywords: railborne, pseudo-turntable imaging, inverse synthetic aperture radar, radar targets, stealth performance

\section{Introduction}

Some special targets are required to have the stealth ability against radars in the procedure of design, such as military aircrafts and missiles; it is generally desired to obtain small RCS in some polarization mode when they are flying towards radars [1]. RCS reduction techniques mainly include material selection and shape designs $[2,3,6]$. It is a basic electromagnetic scattering method to measure the overall RCS of the targets directly, but this approach has visible disadvantage. Just like that signal spectrum can not express the time-varying characteristics of frequency; overall RCS can not characterize spacevarying characteristics of echo power, so that it is not clear which parts or components of the targets have maximum contributions to the echo power at an assigned perspective according to overall RCS. Hence, we are unable to take measurements to reduce the RCS efficiently [1-3]. An ingenious method is proposed here, i.e., not only measuring the overall RCS of all aspects, but also measuring high resolution two-dimensional projection of extended targets at all perspectives in the radar imaging plane. Therefore, it is of great significance to use the twodimensional imaging technology to measure the stealth performance of the targets against radars [4].

As to small targets, they can be put on a turntable, and the images at different azimuths can be obtained without moving the measurement system. We also could get the images at different elevations by adjusting the height of the turntable or the height of the measurement system, so that we could obtain full-perspective measurement on stealth performance [3-5]. Echo acquisition can not only be dynamic (the target moves with an uniform angular velocity on the turntable), but also can be static (targets remain stationary at an observation angle first, after echoes acquisition are completed, the targets move to the next visual angle with the same step). Such measurements can be performed in an anechoic chamber or on an outdoor field; the turntable motion model is adopted for radar imaging. Since the target to be detected (TBD) is cooperative, the two dimensional radar images can be obtained directly using the range-Doppler algorithm (RDA) or polar format algorithm (PFA) [7-8].

However, as to heavy targets (e.g., up to 30 tons), it is difficult to revolve the target on the turntable. In this paper, a method called "railborne pseudo-turntable imaging" is proposed, which can realize the stealth performance measurement of heavy targets on outdoor field.

\section{Railborne pseudo-turntable imaging Measurement scheme}

The heavy targets remain stationary in this scenario; the imaging measurement for the targets to be detected in different perspectives is achieved when the measurement system glides through the closed rail. The scenario is shown in Fig. 1.

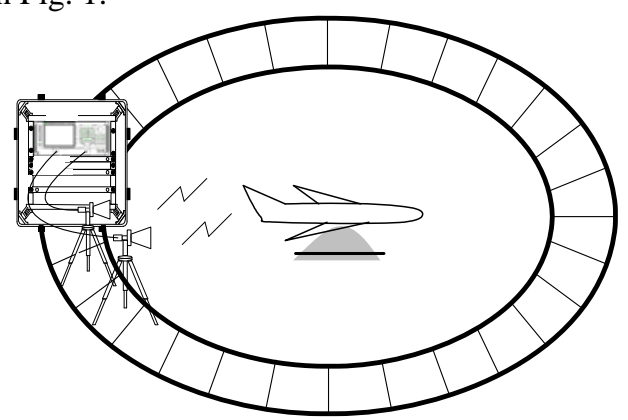

Fig. 1. Railborne pseudo-turntable imaging measurement scenario

The shape of the rail can be designed according to the 
field, which means that the shape of the rail does not have to be a standard circle. Actually, due to the inevitable error, an ideal circle can never be obtained, even if it is constructed according to the blueprint of a circular rail. The target to be detected could be regarded as a pseudocooperative target owing to the non-ideal circular rail. The distance between the measurement system and the center of the target is changed during a full observation period for one inverse synthetic aperture radar (ISAR) image, which is equivalent to slight translation in ISAR imaging called pseudo-turntable motion. If the distances between each point on the track and the targets can be measured accurately using other instrument, we can use the distance information to process radial range alignment and phase correction at different perspectives, which are called motion compensation. After that, the target's motion can be regarded as an ideal turntable motion approximately, and at last the two-dimensional imaging measurement is achieved. In addition, the power attenuation difference caused by the distance difference can be obtained using the radar equation, so we can also realize echo power compensation.

\section{$3 \quad$ Pseudo-turntable imaging algorithm}

The measurement system can transmit wideband signals with frequency scan mode. Electromagnetic waves are illuminated toward the target to be detected, and then the return waves are acquired. Range profile of a certain visual angle can be obtained through a frame of frequency scan. If wideband linear frequency modulated signals are used, range profile of a perspective can be obtained in one pulse [4-5].

The procedures of pseudo-turntable imaging are presented here in detail.

1) Range alignment and phase compensation are performed for the collected radar baseband return wave. The accurate pre-measured distance between the location of measurement facility and the center of the target is necessary.

2) Pulse compression of the baseband return wave is implemented.

3) FFT is operated along the azimuth dimension for each range cell. The modulus of the FFT output is the two-dimensional image.

The complex sampling rate should not less than 1.25 times of the signal bandwidth for the intermediate frequency (IF) analog echoes. The baseband echo signals are acquired after digital down-conversion (DDC) processing.

The first phase compensation factor which should be multiplied with the baseband echo signal is shown as follows

$$
\phi_{c 1}=\exp \left(j 4 \pi R_{i} / \lambda\right)
$$

Where $\lambda$ is the radar wavelength, $R_{i}$ is the radial distance between the target and the electromagnetic scattering measurement system, the subscript $i$ denotes the frame number.

The second phase compensation factor which should be multiplied with the baseband echo signal is shown as follows

$$
\phi_{c 2}=\exp \left\{j 2 \pi f_{m} R_{i} / c\right\}
$$

Where $f_{m}$ demotes the center frequency of radar transmitted signal, and $c$ is the speed of light.

Actually, the two phase compensation operations can be combined in one operation. The total phase compensation factor is shown as follows

$$
\phi_{c}=\exp \left\{j\left(2 \pi f_{m} R_{i} / c+4 \pi R_{i} / \lambda\right)\right\}
$$

Transforming the phase-compensated digital baseband echoes into frequency domain using FFT, and then multiplying it by range compensation factor, which is shown as follows

$$
R_{c}=\exp \left\{j 2 \pi f\left(\tau_{i}-\tau_{n}\right)\right\}
$$

Where, $\tau_{i}=2 R_{i} / c, f$ is the corresponding frequency along radial range dimension.

The echoes of all frames are aligned to Frame $n$ after range compensation; $n$ could be assigned to any one from 1 to $M$.

The range-aligned baseband echoes are multiplied by the matching reference signal in frequency domain, and IFFT is performed to the output of product in range dimension to get the one-dimensional range profile. The Range-Doppler imaging is completed after iterating the above operations for each frame of echoes, and operating FFT to total $M$ range profiles in the cross-range direction. The number of the accumulated imaging frame $M$ could be determined according to the requirement of azimuth resolution.

Fig. 2 shows the flowchart of the pseudo-turntable imaging algorithm. If frequency sweep measurement method is adopted, FFT operation will not be required, since the collected echoes are already in frequency domain. 


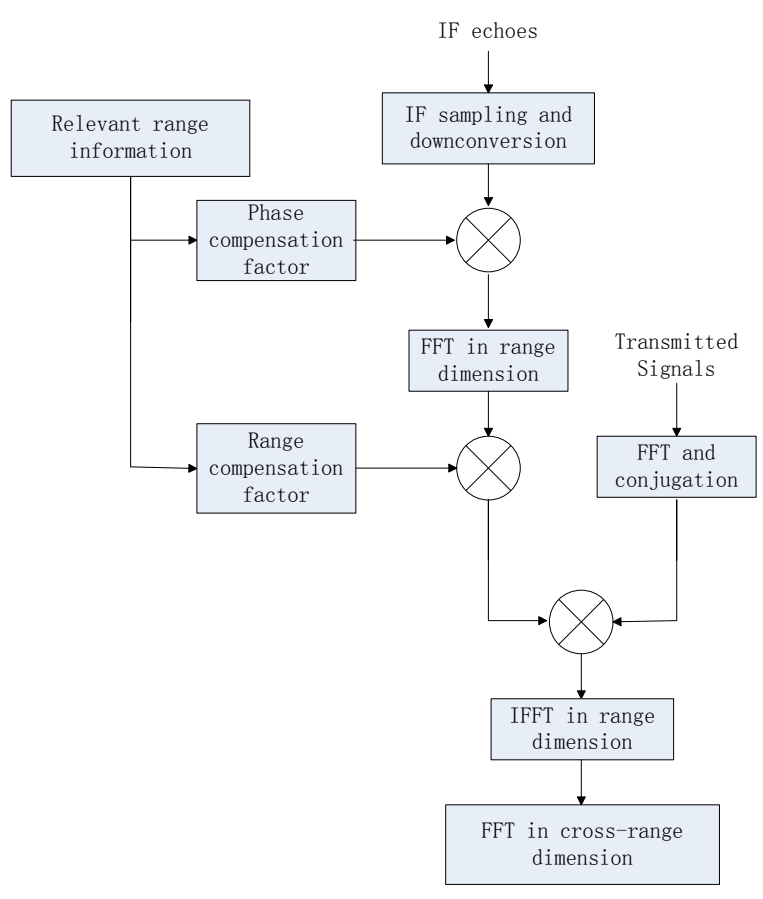

Fig. 2. The flowchart of the pseudo-turntable imaging algorithm

\section{$4 \quad$ Experiments and analysis}

The normal distance between the center of the target to be detected and the antenna of measurement system is $5.69 \mathrm{~m}$; we simulate an irregular rail by adding slight range migration in different perspectives, and then record the distance at each observation position. The measurement system mainly consists of a vector network analyzer, a transmitting antenna, a receiving antenna and remote console. In the experiments, frequency scanning mode is adopted to eradiate electromagnetic signals. The frequency step is $2 \mathrm{MHz}$, the transmitting power is $5 \mathrm{dBsm}$, the gain of both the transmitting antenna and receiving antenna is $19 \mathrm{~dB}$, and the beam width of the antenna is $10^{\circ}$. The RF cable length from the transmitting antenna to the measuring equipment is $2.85 \mathrm{~m}$, so is the receiving antenna. The cable length should be considered for correction in range calibration along down-range dimension. The distance between the transmitting and receiving antenna is $0.36 \mathrm{~m}$.

\subsection{Experiment 1: imaging measurement at 4G- $5 \mathrm{GHz}$}

In this experiment, let the bandwidth to be $1 \mathrm{GHz}$; and the step of the observation angle is $0.2^{\circ}$. The targets are three scattering centers consisting of a rectangular plate ( 1 in Fig. 3), a small metal ball (2 in Fig. 3) and a big metal ball ( 3 in Fig. 3). The distance between target 1 and target 2 , target 2 and target 3 , target 1 and target 3 is $34 \mathrm{~cm}$, $40 \mathrm{~cm}, 50 \mathrm{~cm}$ respectively. The placement of the targets is shown in Fig. 3.

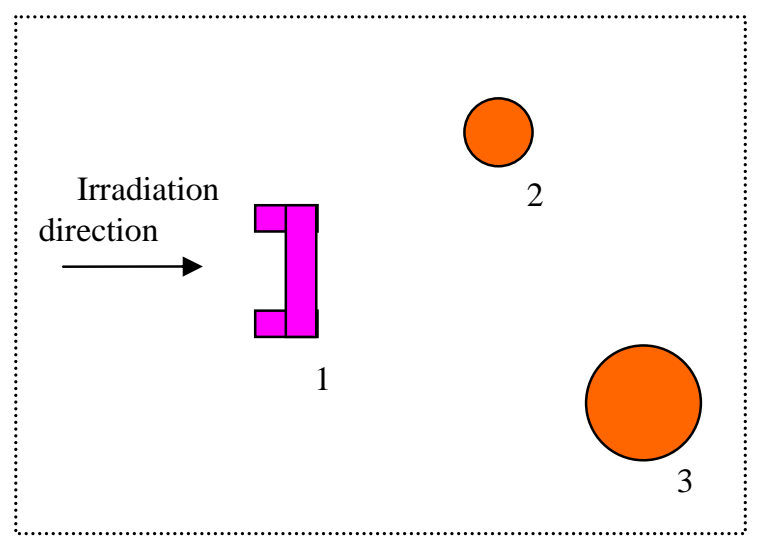

Fig. 3. The placement between scattering centers (4G-5GHz)

The result of pseudo-turntable imaging is shown in Fig. 4. It is clear that the image agrees with the targets' actual placement perfectly. The range resolution is $0.15 \mathrm{~m}$; the imaging accumulated angle is $12^{\circ}$ and the corresponding azimuth resolution is $0.1592 \mathrm{~m}$.

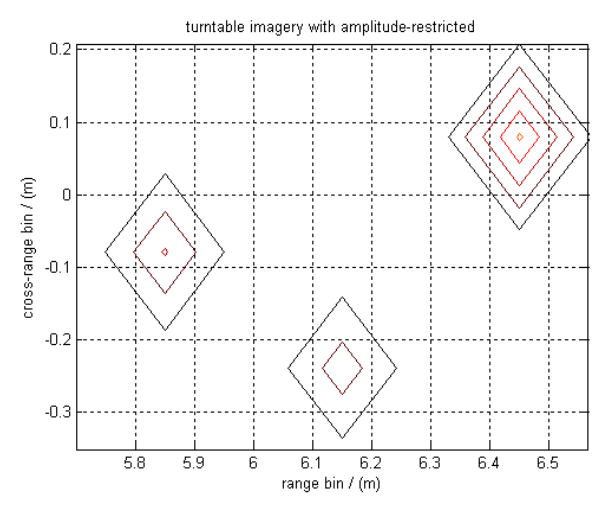

Fig. 4. Result of pseudo-turntable imaging (4G-5GHz)

\subsection{Experiment 2: imaging measurement at 9G- $11 \mathrm{GHz}$}

In this experiment, let the bandwidth to be $2 \mathrm{GHz}$. The step of the observation angle is $0.1^{\circ}$. The targets are three scattering centers consisting of a rectangular plate (1 in Fig. 5), a metal ball (2 in Fig. 5), and a U-shape corner reflector (3 in Fig. 5). The placement of the targets is shown in Fig. 5. 


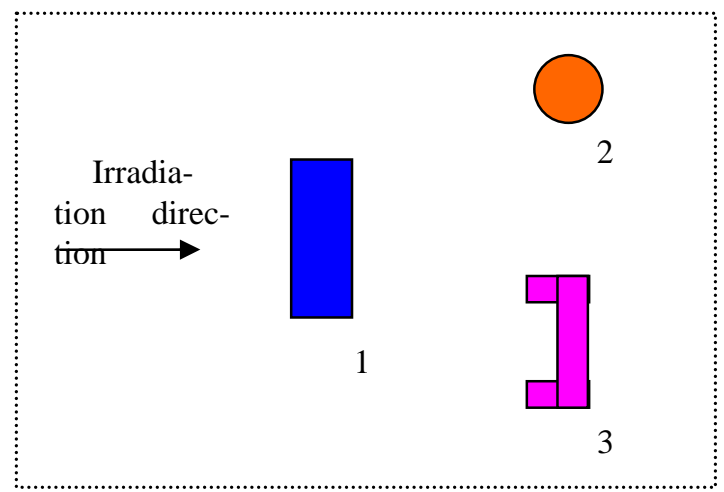

Fig. 5. The placement between scattering centers (9G-11GHz)

Fig. 6 shows the pseudo-turntable image of the collected echoes, which agrees with the targets' actual placement perfectly too. Here the range resolution is $0.075 \mathrm{~m}$; imaging accumulated angle is $12^{\circ}$ and the corresponding azimuth resolution is $0.07162 \mathrm{~m}$.

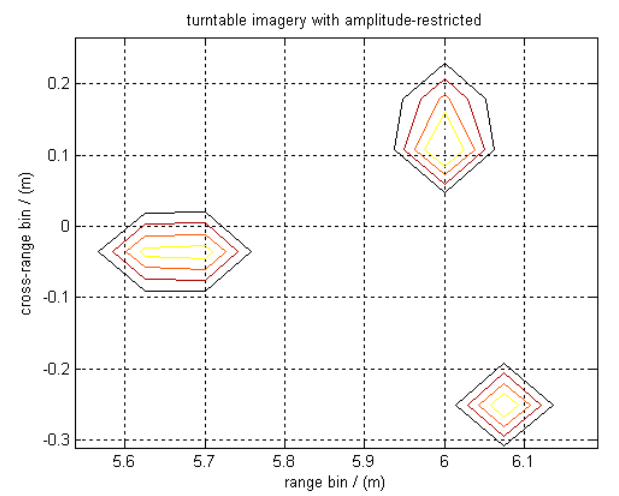

Fig. 6. Result of pseudo-turntable imaging (9G-11GHz)

\section{Conclusions}

Under the scheme that the measurement system glides through a fixed rail, it is feasible to operate pseudoturntable imaging with two-dimensional ISAR imaging theorem. This approach can suit the requirement of stealth performance measurement for radar targets, and guide the implementation of the RCS reduction. In general, only a slight ranging error is existed in this measurement method because the distances could be measured accurately prior to real measuring experiments. When the facility is working while it is gliding through the rail, the non-uniform spatial sampling of the observation perspectives may be existed owing to the non-uniform gliding angular velocity. Fortunately, the fluctuation of the angular velocity can be tolerated if is not more than ten percent of the average angular velocity. Obviously, as to the same linear velocity fluctuation, the larger the distance between rail and the measured target, the smaller the fluctuation of the angular velocity, and the more accurate the measurement. The simulation results show that the approach is effective.

\section{References}

1. Wei Rao, Gang Li, Xiqin Wang, "ISAR imaging of uniformly rotating targets via parametric matching pursuit," IEEE International Geosciences and Remote Sensing Symposium, 2011, pp. $1682-1685$.

2. J. Grajal, B. Mencia-Oliva, O. A. Yeste-Ojeda, A. F. García-Fernández, G. Rubio-Cidre, "A prototype of High Resolution ISAR imaging system at millimeter-wave band," IEEE CIE International Conference, Oct,2011, pp. 551 554.

3. Xiaohui Qiu, Yang Zhao, "An Non-Parametric Rotating Angle Acquisition Method for Optimal ISAR Imaging," Antenna and Propagation Society International Sysposiun, July,2006, pp. $2697-2700$.

4. YiMin Li, Hongyi Liu, "RCS reduction of missible-borne quasi-traveling wave microstrip antenna," Electronic Measurement \& Instruments, ICEMI, Aug,2009, pp. 246 - 249.

5. Yongsheng Xiao, Jianjiang Zhou, Fei Wang, Weiqiang Liu, "Design and Optimization of Radio Frequency Stealth Signal Based on SAGA,” 2010 International Conference on Computer Application and System Modeling, OCT,2010, pp. $580-584$.

6. E. F. Knott, J.F. Shaeffer, and M. T. Tuley, Radar Cross Section, Norwood, MA: Artech House, 1985.

7. Y. Luo, Q. Zhang, C. Qiu , X. Liang and K. Li, "MicroDoppler effect analysis and feature extraction in ISAR imaging with stepped-frequency chirp signals,” IEEE Trans. Geosci. Remote Sens, vol. 48, 2010, pp. 2087 -2098.

8. D. Zhu, L. Wang, Y. Yu, Q. Tao and Z. Zhu "Robust ISAR range alignment via minimizing the entropy of the average range profile", IEEE Geosci. Remote Sens. Lett, vol. 6, 2009, pp. $204-208$. 